\title{
Existence of a Solution of a Certain Volterra - Fredholm Integro Differential Equations
}

\author{
Akram H. Mahmood \\ Lamyaa H. Sadoon \\ Department of Mathematics / College of Education \\ University of Mosul
}

Received

06 / 03 / 2011
Accepted

06 / 04 / 2011

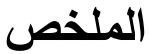

في هذا البحث درسنا وجود الحل الثنامل لمعادلة فولتثرا - فريدهولم التفاضلية التكاملية

غير الخطية مع شرط غير موقعي في فضاء بناخ. النتائج التي تم الحصول عليها اعتمدت على

مبرهنة شاودر - ليري المتتاوبة.
\end{abstract}

\begin{abstract}
In this paper, e investigate the existence of global solution for nonlinear mixed Volterra-Fredholm integro differential equation with nonlocal condition in Banach space.

Our results depend on Leray -Schauder alternative theorem.
\end{abstract}

Keywords: Existence theorem; Global solution; Volterra-Fredholm integro differential equations; Banach space; Leray -Schauder alternative.

\section{Introduction}

Many authors [4,5,6] have interested to solve the linear and nonlinear integral and integro differential equations of type Volterra and Volterra- Fredholm, for example in [7], Pachpatte studied the existence and uniqueness of solutions of mixed Volterra- Fredholm type integral equations which a rises in various physical and biological problems in [8], Pachpatte studied the problem of global existence of solutions of some Volterra integro differential equations, also in [2], Balachandran and Kanagrajan studied the problems of existence and uniqueness of solutions of a class of nonlinear fuzzy Volterra- Fredholm integral equations, in [3], El-Borai and Abdou considered the existence and uniqueness of solutions of nonlinear integral equations of the second kind of type Volterra- Fredholm.Also the normality and continuity of the integral operator are discussed, in [10], Tidke investigated the existence 
of global solutions to first order initial -value problems with nonlocal condition for nonlinear mixed Volterra- Fredholm integro differential equations in Banach space. Finally in [1], Balachandran and Kim established sufficient conditions for the existence and uniqueness of random solutions of nonlinear Volterra-Fredholm stochastic integral equations of mixed type by using admissibility theory and fixed point theorem.

The purpose of this paper is to study the basic problem of existence of global solutions of nonlinear integro-differential equation of VolterraFredholm integral equation of the form

$$
\begin{gathered}
x^{\prime}(t)=f\left(t, x(t), \int_{o}^{t} k(t, s) G(s, x(s)) d s, \int_{o}^{b} h(t, s) Q(s, x(s)) d s\right) \\
x(0)+u(x)=x_{0}
\end{gathered}
$$

By using theorem known as Leray - Schauder alternative.

Where $f:[0, b] \times R^{n} \times R^{n} \times R^{n} \rightarrow R^{n}$ is a function, $k, h:[0, b] \times[0, b] \rightarrow R^{n}$ are continuous functions and $G, Q:[0, b] \times R^{n} \rightarrow R^{n}$ are also continuous functions and $u: B \rightarrow R^{n}$ is given function and $x_{0}$ is given element of $R^{n}$ and $R^{n}$ denotes the Euclidean n-space with norm $\|$.$\| . and let$ $B=c\left([0, b], R^{n}\right)$ be the Banach space of all continuous functions from [0,b] into $R^{n} \quad$ endowed with supremum norm $\|x\|_{B}=\sup \{\|x(t)\|: t \in[0, b]\}$.

The paper is organized as follows: In section 2, we present the preliminaries and hypotheses. And, in Section 3, we deal with the main results.

\section{Preliminaries and Hypotheses}

Our existence theorem are based on the following theorem, which is a version of the topological transversality theorem given by Tidke [10] and is known as Leray - Schauder alternative.

\section{Theorem (2.1) [10] (Leray -Schauder alternative)}

Let $\mathrm{S}$ be a convex subset of a normed linear space $\mathrm{E}$ and assume $0 \in S$. Let $F: S \rightarrow S$ be a completely continuous operator and let $\varepsilon(F)=\{x \in S: x=\lambda F x$ for some $0<\lambda<1\}$, then either $\varepsilon(F)$ is unbounded or $\mathrm{F}$ has a fixed point.

\section{Theorem (2.2) [9] (The Arzela - Ascoli Theorem)}

Let $\mathrm{A}$ be a subset of $(\varphi(I), \rho)$. Then the following statements are equivalent: 
(i) A is compact.

(ii) $\mathrm{A}$ is closed subset of $(\varphi(I), \rho)$ and is both uniformly bounded and equicontinuous over I.

We list the following hypotheses used in our discussion.

1) There exists a continuous function $p:[0, b] \rightarrow R_{+}=[0, \infty)$ and there exists a constant $L \geq 0$, s.t. $\|k(t, s)\| \leq L$ for $t \geq s \geq 0$ and $\| \int_{0}^{t} k(t, s) G(s,(x(s)) d s \quad \| \leq L p(t) H(\|x\|) \quad$ for every $t, s \in[o, b] \quad$ and $x \in R^{n}$, where $H: R_{+} \rightarrow(0, \infty)$ is a continuous nondecreasing function.

2) There exists a continuous function $q:[0, b] \rightarrow R_{+}=[0, \infty)$ and a constant $\quad N \geq 0 \quad$ s.t. $\quad\|h(t, s)\| \leq N, \quad$ for $t \geq s \geq 0 \quad$ and $\left\|\int_{0}^{b} h(t, s) Q(s, x(s)) d s\right\| \leq N q(t) M(\|x\|)$ for every $t, s \in[o, b] \quad$ and $x \in R^{n}$, where $M: R_{+} \rightarrow(0, \infty)$ is a continuous nondecreasing function.

3) There exists a continuous function $l:[0, b] \rightarrow R_{+}=[0, \infty)$ s.t. $\|f(t, x, y, z)\| \leq l(t)(\|x\|+\|y\|+\|z\|)$ for every $t \in[o, b]$ and $x, y, z \in R^{n}$.

4) There exists a constant $A$ s.t. $\|u(x)\| \leq A$, for $x \in R^{n}$..

\section{Main results}

The following theorem ensures the existence of solution to equation (1.1)-(1.2).

\section{Theorem (3.1)}

If the hypotheses (1-4) are satisfied, then the initial -value problem (1.1)-(1.2) has a solution $\mathrm{x}$ defined on $[0, \mathrm{~b}]$ provided $\mathrm{b}$ satisfies

$\int_{0}^{b} 3 R(s) d s<\int_{c}^{\infty} \frac{d s}{s+H(s)+M(S)}$

Where $c=\left\|x_{0}\right\|+A$ and $R(t)=\max \{l(t), L p(t), N q(t)\}$ for $t \in[o, b]$.

\section{Proof}

\section{Step I}

The proof will be given in the three steps.

To use theorem (2.1), we establish the priori bounds on the solution of the problem

$$
x^{\prime}(t)=\lambda f\left(t, x(t), \int_{o}^{t} k(t, s) G(s, x(s)) d s,{ }_{o}^{b} h(t, s) Q(s, x(s)) d s\right), \underset{t \in[0, b]}{,}
$$

Under the initial condition (1.2) for $\lambda \in(0,1)$. Let $x(t)$ be a solution of the problem (3.2)-(1.2), then it satisfies the equivalent integral equation 
$x(t)=x_{0}-u(x)+\lambda \int_{0}^{t} f\left(s, x(s), \int_{o}^{s} k(s, \tau) G(\tau, x(\tau)) d \tau, \int_{o}^{b} h(s, \tau) Q(\tau, x(\tau)) d \tau\right) d s$

for $t \in[0, b]$, from (3.3) and using the hypotheses (1-4) and the fact that $\lambda \in(0,1)$, we have

$$
\begin{gathered}
\|x(t)\| \leq\left\|x_{0}-u(x)\right\|+\| \int_{0}^{t} f\left(s, x(s), \int_{o}^{s} k(s, \tau) G(\tau, x(\tau)) d \tau,\right. \\
\left.\quad \int_{o}^{b} h(s, \tau) Q(\tau, x(\tau)) d \tau\right) d s \| \\
\leq\left\{\left\|x_{0}\right\|+A\right\}+\int_{0}^{t} \| f\left(s, x(s), \int_{o}^{s} k(s, \tau) G(\tau, x(\tau)) d \tau,\right. \\
\left.\quad \int_{o}^{b} h(s, \tau) Q(\tau, x(\tau)) d \tau\right) \| d s \\
\leq\left\{\left\|x_{0}\right\|+A\right\}+\int_{0}^{t} l(s)(\|x(s)\|+\operatorname{Lp}(s) H(\|x(s)\|+N q(s) M(\|x(s)\|) d s
\end{gathered}
$$

Let $\mathrm{v}(\mathrm{t})$ be defined by the right hand side of (3.4), then

$$
\begin{gathered}
\|x(t)\| \leq v(t) \quad v(0)=\left\{\left\|x_{0}\right\|+A\right\}=c \quad \text { and } \\
v(t)=\left\{\left\|x_{0}\right\|+A\right\}+\int_{0}^{t} l(s)(\|x(s)\|+\operatorname{Lp}(s) H(\|x(s)\|)+N q(s) M(\|x(s)\|)) d s
\end{gathered}
$$

then

$v(t)=c+\int_{0}^{t} l(s)(\|x(s)\|+\operatorname{Lp}(s) H(\|x(s)\|)+N q(s) M(\|x(s)\|)) d s$

Differentiating both sides of (3.5) with respect to $t$ we have

$$
\begin{aligned}
& v^{\prime}(t) \leq l(t)(v(t)+L P(t) H(v(t))+N q(t) M(v(t))) \\
& v^{\prime}(t) \leq 3 R(t)(v(t)+H(v(t))+M(v(t)))
\end{aligned}
$$

That is,

$$
\frac{v^{\prime}(t)}{v(t)+H(v(t))+M(v(t))} \leq 3 R(t)
$$

Integrating of (3.7) from ( 0 to $t)$, the change of variable and the condition (3.1) gives

$$
\int_{c}^{v(t)} \frac{d s}{s+H(s)+M(s)} \leq \int_{0}^{t} 3 R(s) d s \leq \int_{0}^{b} 3 R(s) d s<\int_{c}^{\infty} \frac{d s}{s+H(s)+M(s)}
$$

From this inequality, we conclude that, there is a constant $\alpha$ independent of $\lambda \in(0,1)$ such that $v(t) \leq \alpha$ and hence $\|x(t)\| \leq \alpha$ for $t \in[0, b]$, and consequently $\|x\|_{B}=\sup \{\|x(t)\|: t \in[0, b]\} \leq \alpha$. 


\section{Step II}

We define $B=c\left([o, b], R^{n}\right)$ to be the Banach space of all continuous functions from [0,b] into $R^{n}$ endowed with sup norm defined above. We rewrite the problem (1.1)-(1.2) as follows.

If $y \in B$ and $x(t)=x_{0}-u(x)+y(t), t \in[0, b]$ it is easy to see that $\mathrm{y}$ satisfies $y(0)=y_{0}=0$

$$
\begin{aligned}
y(t)=\int_{0}^{t} f\left(s, y(s)+x_{0}-u(y), \int_{0}^{s} k(s, \tau) G\left(\tau, y(\tau)+x_{0}-u(y)\right) d \tau,\right. \\
\left.\int_{0}^{b} h(s, \tau) Q\left(\tau, y(\tau)+x_{0}-u(y)\right) d \tau\right) d s \quad, t \in[0, b]
\end{aligned}
$$

If and only if $\mathrm{x}(\mathrm{t})$ satisfies

$x(t)=x_{0}-u(x)+\int_{0}^{t} f\left(s, x(s), \int_{0}^{s} k(s, \tau) G(\tau, x(\tau)) d \tau, \int_{0}^{b} h(s, \tau) Q(\tau, x(\tau)) d \tau\right) d s$,

$$
t \in[0, b]
$$

We define the operator $F: B_{0} \rightarrow B_{0}, B_{0}=\{y \in B: y(0)=0\}$ by

$$
\begin{aligned}
(F y)(t)= & \int_{0}^{t} f\left(s, y(s)+x_{0}-u(y), \int_{0}^{s} k(s, \tau) G\left(\tau, y(\tau)+x_{0}-u(y)\right) d \tau,\right. \\
& \left.\int_{0}^{b} h(s, \tau) Q\left(\tau, y(\tau)+x_{0}-u(y)\right) d \tau\right) d s \quad, t \in[0, b]
\end{aligned}
$$

Then $\mathrm{F}$ is clearly continuous.

Next, we prove that $\mathrm{F}$ is completely continuous.

Let $\left\{b_{m}\right\}$ be a bounded sequence in $B_{0}$, i.e. $\left\|b_{m}\right\|_{B} \leq \delta$ for all $m$, where $\delta$ is a positive constant. From (3.9) and using the hypotheses (1-4) and letting $R^{*}=\sup \{3 R(t): t \in[0, b]\}$ we have

$$
\begin{aligned}
\left\|\left(F b_{m}\right)(t)\right\| \leq & \int_{0}^{t} \| f\left(s, b_{m}(s)+x_{0}-u\left(b_{m}\right), \int_{0}^{s} k(s, \tau) G\left(\tau, b_{m}(\tau)\right)+x_{0}-u\left(b_{m}\right)\right) d \tau \\
& \int_{0}^{b} h(s, \tau) Q\left(\tau, b_{m}(\tau)+x_{0}-u\left(b_{m}\right)\right) d \tau \| d s \\
& \leq \int_{0}^{t} l(s)(\delta+c+L p(s) H(\delta+c)+N q(s) M(\delta+c)) d s \\
& \leq \int_{0}^{t} R^{*}\left(\delta+c+R^{*} H(\delta+c)+R^{*} M(\delta+c)\right) d s \\
& \leq b R^{*}\left(\delta+c+R^{*} H(\delta+c)+R^{*} M(\delta+c)\right)
\end{aligned}
$$

This means that $\left\{F b_{m}\right\}$ is uniformly bounded.

\section{Step III}

We shall show that the sequence $\left\{F b_{m}\right\}$ is equicontinuous Let $0 \leq t_{1} \leq t_{2} \leq b$. Then from (3.9) and using the hypotheses (1-4) and letting $R^{*}=\sup \{3 R(t): t \in[0, b]\}$ we have 


$$
\begin{gathered}
\left\|F b_{m}\left(t_{2}\right)-F b_{m}\left(t_{1}\right)\right\| \leq \int_{t_{1}}^{t_{2}} \| f\left(s, b_{m}(s)+x_{0}-u\left(b_{m}\right), \int_{0}^{s} k(s, \tau) G\left(\tau, b_{m}(\tau)+x_{0}-u\left(b_{m}\right) d \tau,\right.\right. \\
\int_{0}^{b} h(s, \tau) Q\left(\tau, b_{m}(\tau)+x_{0}-u\left(b_{m}\right)\right) d \tau \| d s \\
\leq \int_{t_{1}}^{t_{2}} l(s)\left(\left\|b_{m}(s)+x_{0}-u\left(b_{m}\right)\right\|\right)+L p(s) H\left(\left\|b_{m}(s)+x_{0}-u\left(b_{m}\right)\right\|\right)+ \\
\quad N q(s) M\left(\left\|b_{m}(s)+x_{0}-u\left(b_{m}\right)\right\|\right) d s
\end{gathered}
$$

From (3.11) we conclude that $\left\{F b_{m}\right\}$ is equicontinuous and hence by the Arzela- Ascoli theorem (2.2) the operator $\mathrm{F}$ is completely continuous.

Finally, the set $\varepsilon(F)=\left\{y \in B_{0}, y=\lambda F y, \lambda \in(0,1)\right\}$ is bounded in $\mathrm{B}$, since for every $y \in \varepsilon(F)$, the function $x(t)=x_{0}-u(x)+y(t)$ is a solution of (3.2)-(1.2) for which we have provid that $\|x\|_{B} \leq \alpha$ and hence $\|y\|_{B} \leq \alpha+c$. Consequently, by theorem (2.1), the operator $\mathrm{F}$ has a fixed point in $B_{0}$. This means that the initial - value problem (1.1)-(1.2) has a solution. This completely the proof of the theorem.

\section{References}

1) K.Balachandran and J.H.Kim, "Existence of solutions of nonlinear stochastic Volterra-Fredholm integral equations of mixed type", J.Math. Sci., Vol. (2010), p(1-16), (2010).

2) K.Balachandran and K.Kanagarajan," Existence of solutions of general nonlinear Fuzzy Volterra-Fredholm integrale Vol. (2005), No.(3), p(333-343), (2005).

3) M.M.EL-Boria, M.A.Abdou and M.M.EL-Kojok, "On a discussion of nonlinear integral equation of type Volterra-Fredholm", J.Ksiam, Vol.(10), No.(2), p(59-83), (2006).

4) N.Lungu and I.A.Rus, "On a functional Volterra-Fredholm integral equations Via - Picard operator", J.Math. Ieq., Vol.(3), No.(4), p(519-527), (2009).

5) D.Margetis and J.Choi, "Generalized iteration method for first - kind integral equations", J.Appl. Math. Vol.(117), p(1-25), (2006).

6) T.L.Nguyan and T.D.Bui, "A nonlinear wave equations with a nonlinear integral equations involving the boundary value", J.Elec.Diff.Eqs., Vol.(2004), No.(103), p(1-21), (2004).

7) B.G.Pachpatte, "On mixed Volterra-Fredholm type integral equations", J.Indian.pure appl.Math.,17(4), p (488-496), (1986).

8) B.G.Pachpatte, "Applications of the Leray - Schuder of the alternative to some Volterra integral and integro differential equations", J.Indian.pure appl.Math., 26(12), p (1161-1168), (1995).

9) C.G.C.Pitts, "Introduction to metric spaces", Oliver and Boyd, Edinburgh, (1972)

10) H.L.Tidke, "Existence of global solutions to nonlinear mixed Volterra-Fredholm integro differential equations with nonlocal conditions", J.elec.Diff.Eqs., Vol.(2009), No.(55), p (1- 7), (2009). 